\title{
Collective Memory and Spatial Sorting in Animal Groups
}

\author{
Iain D. Couzin*†, Jens Krause $\dagger$, Richard James $\$$, Graeme D. Ruxton§ \\ AND NigEL R. FRANKS $\Phi$ \\ $\dagger$ Centre for Biodiversity and Conservation, School of Biology, University of Leeds, Leeds LS2 9JT, \\ U.K., $\ddagger$ Department of Physics, University of Bath, Bath BA2 7AY, U.K., §Division of Environmental \\ and Evolutionary Biology, Graham Kerr Building, University of Glasgow, Glasgow G12 8QQ, U.K. and \\ - Centre for Behavioural Biology, School of Biological Sciences, University of Bristol, \\ Bristol BS8 $1 U G, U . K$.
}

(Received on 15 January 2002, Accepted in revised form on 19 April 2002)

\begin{abstract}
We present a self-organizing model of group formation in three-dimensional space, and use it to investigate the spatial dynamics of animal groups such as fish schools and bird flocks. We reveal the existence of major group-level behavioural transitions related to minor changes in individual-level interactions. Further, we present the first evidence for collective memory in such animal groups (where the previous history of group structure influences the collective behaviour exhibited as individual interactions change) during the transition of a group from one type of collective behaviour to another. The model is then used to show how differences among individuals influence group structure, and how individuals employing simple, local rules of thumb, can accurately change their spatial position within a group (e.g. to move to the centre, the front, or the periphery) in the absence of information on their current position within the group as a whole. These results are considered in the context of the evolution and ecological importance of animal groups.
\end{abstract}

(C) 2002 Elsevier Science Ltd. All rights reserved.

\section{Introduction}

Many animal groups such as fish schools and bird flocks clearly display structural order, with the behaviour of the organisms so integrated that even though they may change shape and direction, they appear to move as a single coherent entity. Many of the collective behaviours exhibited by such groups can only be understood by considering the very large number of interactions among group members. Individual-based computer simulations are a very useful analytical tool to study such groups, and

*Corresponding author.

E-mail address: iaincouzin@hotmail.com (I.D. Couzin). using this technique, it has been possible to demonstrate that group leadership, hierarchical control, and global information are not necessary for collective behaviour (Aoki, 1982; Reynolds, 1987; Huth \& Wissel, 1992; Gueron et al., 1996; Czirók et al., 1997, 1999; Warburton, 1997; Helbing et al., 2000). The simplest models of collective motion merely assume that individuals move at a constant speed and assume the average direction of motion (this direction being subject to error) of those within a local neighbourhood (Czirók et al., 1997, 1999). Such models are useful since their minimalism allows them to be analysed using techniques developed for non-equilibrium physical systems (for a 
review, see Czirók \& Vicsek, 2001). This comes at the cost of biological realism, however. For example, the particles neither avoid collisions nor exhibit attraction towards others. Consequently, they cannot form a self-bounded group when error is greater than zero, and thus these models cannot fully explain the clearly defined animal groups seen in many species.

Here, we use a more biologically realistic (yet still simple) model of aggregation behaviour that is based upon an abstraction of aggregation tendencies evident in biological systems (Partridge \& Pitcher, 1980; Partridge, 1982; Heppner, 1997). Following the approach of Aoki (1982), Reynolds (1987) and Huth \& Wissel (1992), we simulate the behaviour of individuals as resulting from local repulsion, alignment and attractive tendencies based upon the position and orientation of individuals relative to one another. In such models the individual behaviour results in group formation and cohesion, rather than fixing individual density within a periodic domain (as in the simplest models described above). Our simulation exhibits characteristic collective behaviours, similar to those of natural groups, when certain parameters are changed, and we discuss how these different types of collective behaviour are likely to influence the fitness of individuals within such groups. We also use the model to reveal for the first time a novel form of collective memory, where the previous history of the group structure influences collective behaviours as individual interactions change, even though the individuals have no knowledge of what that history is. We hypothesize that this hysteresis phenomenon may be an as yet unexplored property of real animal groups, and discuss the relevance of this finding to our understanding of the evolution of behaviour in grouping organisms.

The spatial structuring within groups has important ecological and evolutionary consequences (Okubo, 1980; Krause \& Ruxton, 2002). Despite this, the majority of previous studies have failed to consider that the costs and benefits of group living are dependent on the spatial positions individuals adopt (see, Krause, 1994, for a review). Group members are not intrinsically identical (Pitcher et al., 1985; Parrish, 1989; DeBlois \& Rose, 1996; Krause et al., 1996) and differences (e.g. behavioural state, age) are likely to influence the positions adopted by individuals within the group. In our model, we investigate how physical and/or motivational differences among individuals may influence the spatial positions of individuals within animal aggregations, and discuss how the results of the model may allow insight into the structuring seen within natural animal aggregates.

\section{The Model}

BEHAVIOURAL RULES: SUMMARY

(1) Individuals attempt to maintain a minimum distance between themselves and others at all times. This rule has the highest priority and corresponds to a frequently observed behaviour of animals in nature (Krause \& Ruxton, 2002). (2) If individuals are not performing an avoidance manoeuvre (rule 1) they tend to be attracted towards other individuals (to avoid being isolated) and to align themselves with neighbours (Partridge \& Pitcher, 1980; Partridge, 1982). These behavioural tendencies are simulated using local perception and simple response behaviours.

\section{BEHAVIOURAL RULES: DESCRIPTION}

$N$ individuals $(i=1,2, \ldots, N)$ with position vectors $\mathbf{c}_{i}$, and unit direction vectors $\mathbf{v}_{\boldsymbol{i}}$ are simulated in continuous three-dimensional space. Time is partitioned into discrete time steps $t$ with a regular spacing $\tau$. Here, $\tau$ is set to $0.1 \mathrm{~s}$, corresponding to the response latency of fish (Partridge \& Pitcher, 1980). In each time step, individuals assess the position and/or orientation of $n$ neighbours within three nonoverlapping behavioural zones (described below; Fig. 1). This information is used to determine a desired direction for each individual for the successive time step $\mathbf{d}_{i}(t+\tau)$ using the following rules.

Each individual attempts to maintain a minimum distance from others within a "zone of repulsion" (zor), modelled as a sphere, centred on the individual, with radius $r_{r}$. If $n_{r}$ neighbours are present in the zor at time $t$, individual $i$ responds by moving away from neighbours 


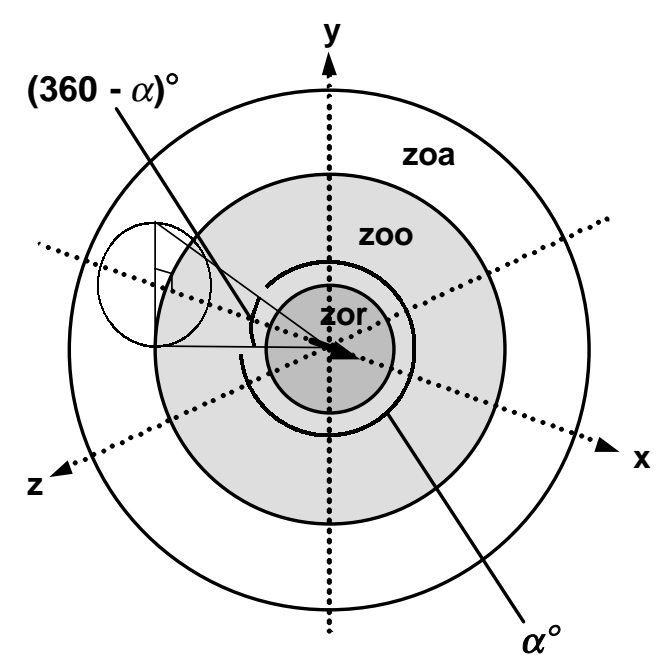

FIG. 1. Representation of an individual in the model centred at the origin: $z o r=$ zone of repulsion, $z o o=$ zone of orientation, $z o a=$ zone of attraction. The possible "blind volume" behind an individual is also shown. $\alpha=$ field of perception.

within this zone:

$$
\mathbf{d}_{r}(t+\tau)=-\sum_{j \neq i}^{n_{r}} \frac{\mathbf{r}_{i j}(t)}{\left|\mathbf{r}_{i j}(t)\right|}
$$

where $\mathbf{r}_{i j}=\left(\mathbf{c}_{j}-\mathbf{c}_{i}\right) /\left|\left(\mathbf{c}_{j}-\mathbf{c}_{i}\right)\right|$ is the unit vector in the direction of neighbour $j$. Note that $s \tau<r_{r}$ avoids singularities in eqn (1). This behavioural rule has the highest priority in the model, so that if $n_{r}>0$, the desired direction $\mathbf{d}_{i}(t+\tau)=\mathbf{d}_{r}(t+$ $\tau)$. The zone of repulsion can be interpreted as individuals maintaining personal space, or avoiding collisions.

If no neighbours are within the zone of repulsion $\left(n_{r}=0\right)$, the individual responds to others within the "zone of orientation" (zoo) and the "zone of attraction" (zoa). These zones are spherical, except for a volume behind the individual within which neighbours are undetectable. This "blind volume" is defined as a cone with interior angle $(360-\alpha)^{\circ}$, where $\alpha$ is defined as the field of perception (see Fig. 1). An individual with $\alpha=360^{\circ}$ can respond to others in any direction within the behavioural zones.

The zone of orientation contains $n_{\mathrm{o}}$ detectable neighbours with $r_{r} \leqslant\left|\left(\mathbf{c}_{j}-\mathbf{c}_{i}\right)\right|<r_{o}$ and the zone of attraction $n_{o}$ detectable neighbours with $r_{o} \leqslant\left|\left(\mathbf{c}_{j}-\mathbf{c}_{i}\right)\right| \leqslant r_{a}$. The widths of these zones are defined as $\Delta r_{o}=r_{o}-r_{r}$ and $\Delta r_{a}=r_{a}-r_{o}$.
An individual will attempt to align itself with neighbours within the zone of orientation, giving

$$
\mathbf{d}_{o}(t+\tau)=\sum_{j=1}^{n_{o}} \frac{\mathbf{v}_{j}(t)}{\left|\mathbf{v}_{j}(t)\right|}
$$

and towards the positions of individuals within the zone of attraction

$$
\mathbf{d}_{a}(t+\tau)=\sum_{j \neq i}^{n_{a}} \frac{\mathbf{r}_{i j}(t)}{\left|\mathbf{r}_{i j}(t)\right|} .
$$

The attraction represents the tendency of organisms to join groups and to avoid being on the periphery, whereas the orientation allows collective movement by minimizing the number of collisions between individuals. If neighbours are only found in the zoo $\left(n=n_{o}\right)$, then $\mathbf{d}_{i}(t+\tau)=$ $\mathbf{d}_{o}(t+\tau)$; likewise if all neighbours are in the zoa $\left(n=n_{a}\right)$, then $\mathbf{d}_{i}(t+\tau)=\mathbf{d}_{a}(t+\tau)$. If neighbours are found in both zones, then $\mathbf{d}_{i}(t+\tau)=$ $\frac{1}{2}\left[\mathbf{d}_{o}(t+\tau)+\mathbf{d}_{a}(t+\tau)\right]$. In the eventuality that the social forces result in a zero vector, or if no individuals are detected, then $\mathbf{d}_{i}(t+\tau)=\mathbf{v}_{i}(t)$.

Decision making in animals is subject to stochastic effects (e.g. sensory error, movement error). This is simulated by modifying $\mathbf{d}_{i}(t+\tau)$ by rotating it by an angle taken at random from a spherically wrapped Gaussian distribution with standard deviation, $\sigma$ (Table 1).

After the above process has been performed for every individual they turn towards the direction vector $\mathbf{d}_{i}(t+\tau)$ by the turning rate $\theta$. Provided the angle between $\mathbf{v}_{i}(t)$ and $\mathbf{d}_{i}(t+\tau)$ is less than the maximum turning angle $\theta \tau$, then $\mathbf{v}_{i}(t+\tau)=\mathbf{d}_{i}(t+\tau)$; if not, the individual rotates by $\theta \tau$ towards the desired direction. To simplify the analysis of parameter space initially we assume that individuals move at a constant speed of $s$ units per second (we investigate the importance of differences in individual speed below). Following these rules individual trajectories can be integrated over time to explore how the behavioural responses influence collective behaviour.

\section{ANALYSIS OF THE MODEL}

To analyse the collective behaviour of the model, we explore the consequences of changing 
TABLE 1

Summary of model parameters. The use of "units" relates to the nondimensionality of certain parameters in the model with the characteristic length scale being associated with the particular organism in question, e.g. $r_{r}$ may be very small for an insect, and the rest of the model parameters can be scaled appropriately

\begin{tabular}{llll}
\hline Parameter & Unit & Symbol & Values explored \\
\hline Number of individuals & None & $N$ & $10-100$ \\
Zone of repulsion & Units & $r_{r}$ & 1 \\
Zone of orientation & Units & $\Delta r_{o}\left(r_{o}-r_{r}\right)$ & $0-15$ \\
Zone of attraction & Units & $\Delta r_{a}\left(r_{a}-r_{o}\right)$ & $0-15$ \\
Field of perception & Degrees & $\alpha$ & $200-360$ \\
Turning rate & Degrees per second & $\theta$ & $10-100$ \\
Speed & Units per second & $s$ & $1-5$ \\
Error (S.D.) & Degrees (rad) & $\sigma$ & $0-11.5(0-0.2 \mathrm{rad})$ \\
Time step increment & Seconds & $\tau$ & 0.1 \\
\hline
\end{tabular}

the values given to parameters (Table 1). Two global properties of the model are calculated from the integrated trajectories of all the individuals; group polarization, $p_{\text {group }}\left[0 \leqslant p_{\text {group }}\right.$ $\leqslant 1$; eqn (4)] and the group angular momentum, $m_{\text {group }}\left[0 \leqslant m_{\text {group }} \leqslant 1\right.$; eqn (5)]. Group polarization increases as the degree of alignment among individuals within the group increases, whereas the group angular momentum is the sum of the angular momenta of the individuals about the centre of the group, $\mathbf{c}_{\text {group }}$ [also known as the group centroid, eqn (6)]. Angular momentum therefore measures the degree of rotation of the group about the group centre. Thus,

$$
\begin{gathered}
p_{\text {group }}(t)=\frac{1}{N}\left|\sum_{i=1}^{N} \mathbf{v}_{i}(t)\right|, \\
m_{\text {group }}(t)=\frac{1}{N}\left|\sum_{i=1}^{N} \mathbf{r}_{i c}(t) \times \mathbf{v}_{i}(t)\right|,
\end{gathered}
$$

where

$$
\mathbf{r}_{i c}=\mathbf{c}_{i}-\mathbf{c}_{\text {group }}
$$

and

$$
\mathbf{c}_{\text {group }}(t)=\frac{1}{N} \sum_{i=1}^{N} \mathbf{c}_{i}(t) .
$$

For each combination of parameters, individuals start with random orientations and at random positions within a sphere in which each can detect at least one individual. Group fragmentation is measured using an extension of the calculation of equivalence classes (Press et al., 1992), where the criterion of interest is the presence of other individuals within the field of perception.

The collective behaviour of the model is analysed after it reaches a dynamically stable state, where the values of the different system measures have stabilized (always within 5000 time steps, equivalent to $8.3 \mathrm{~min}$ of real time, for the range of parameters analysed here).

To understand the influence of individual differences on spatial position within a group, we investigate the consequence of variation in speed $s$, turning rate $\theta$, error $\sigma, r_{r}, r_{o}$ and $r_{a}$ among individuals within a group. To simulate variation, the parameter under investigation is modified by a Gaussian distributed deviate independently drawn for each individual, and is then fixed at that value for the duration of the experiment. The standard deviation of this distribution determines the degree to which individuals differ with respect to that parameter within the group, and is investigated up to a cutoff point beyond which the group tends to fragment. The correlation between these parameters and the distance between individuals and the group centre $\mathbf{c}_{\text {group }}(t)$, and distance to the front of the group, is measured using the Spearman rank correlation coefficient (rho). The front of the group is determined by first 
calculating the direction vector $\mathbf{d}_{\text {group }}(t)$ which extends from the group centre $\mathbf{c}_{\text {group }}(t)$

$$
\mathbf{d}_{\text {group }}(t)=\frac{1}{N} \sum_{i=1}^{N} \mathbf{v}_{i}(t) .
$$

This is then be used to define the plane perpendicular to $\mathbf{d}_{\text {group }}(t)$ and passing through $\mathbf{c}_{\text {group }}(t)$. The individual at the front of the group is that with the greatest minimum distance to the plane on the side to which $\mathbf{d}_{\text {group }}(t)$ extends. The individual at the rear of the group has the greatest minimum distance from the plane on the other side. Other individuals are ranked accordingly (see Fig. 2).

\section{Results}

\section{COLLECTIVE BEHAVIOUR}

As the width of the behavioural zones $\left(\Delta r_{o}\right.$ and $\Delta r_{a}$ ) changes, the collective behaviour of the system exhibits sharp transitions between four

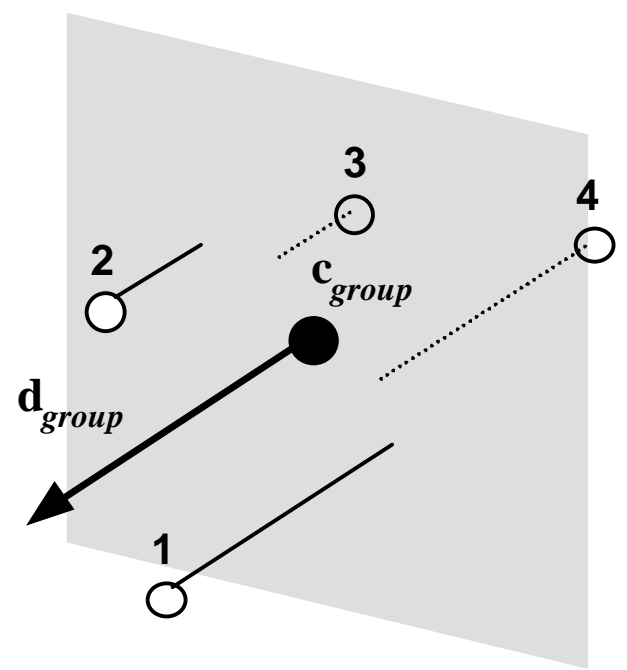

FIG. 2. Measuring the order of four individuals within a hypothetical group from the front. Individuals are represented as points relative to the group centre $\mathbf{c}_{\text {group }}$ with direction $\mathbf{d}_{\text {group }}$. The grey plane is perpendicular to $\mathbf{d}_{\text {group }}$, and passes through the $\mathbf{c}_{\text {group }}$. The shortest line segment between the plane and individuals on the same side of the plane as $\mathbf{d}_{\text {group }}$ are shown as solid lines. These individuals are in the front half of the group. Those on the other side of the plane are shown as dotted lines, and are in the rear half of the group. Note that all lines are parallel with $\mathbf{d}_{\text {group }}$. The length, and direction, of these lines are then used to rank individuals from the front, here shown as 1-4. collective dynamical behaviours (Fig. 3) which we have labelled as follows.

Swarm: an aggregate with cohesion, but a low level of polarization (parallel alignment) among members (low $p_{\text {group }}$ ) and low angular momentum (low $m_{\text {group }}$ ). This occurs when individuals perform attraction and repulsion behaviours, but little or no parallel orientation (Fig. 3(A); $3(\mathrm{E})$ and $(\mathrm{F})$, region a).

Torus: individuals perpetually rotate around an empty core (milling). The direction of rotation is random. $p_{\text {group }}$ is low, but $m_{\text {group }}$ is high. This occurs when $\Delta r_{o}$ is relatively small and $\Delta r_{a}$ is relatively large (Fig. 3(B); 3(E) and (F), region $b)$.

Dynamic parallel group: the group exhibits high $p_{\text {group }}$, but low $m_{\text {group }}$. This type of group is much more mobile than the swarm or torus, and occurs at intermediate values of $\Delta r_{o}$ with intermediate or high values of $\Delta r_{a}$ (Fig. 3(C); (E) and $(\mathrm{F})$, region $\mathrm{c})$.

Highly parallel group: as $\Delta r_{o}$ increases, the group self-organizes into a highly aligned arrangement (very high $p_{\text {group }}$ ) with rectilinear movement (low $m_{\text {group }}$ ) (Fig. 3(D); (E) and (F), region $\mathrm{d})$.

Swarm behaviour is often seen in insects, such as mosquitoes and midges (Okubo \& Chiang, 1974; Ikawa \& Okabe, 1997), and can also be exhibited by fish schools (Pitcher \& Parrish, 1993). The dynamic parallel group exhibits many of the properties associated with aggregations such as bird flocks and fish schools; the individuals are polarized and move as a coherent group, but individuals can move throughout the group and density and group form can fluctuate (Major \& Dill, 1978; Partridge, 1982). The highly parallel group is much more static in terms of exchange of spatial positions within the group than the dynamic parallel group, and the variation in density and form is minimal. The torus formation may seem uncharacteristic of real animal movement within groups. However, this group type is exhibited by natural schools of fish in open water including barracuda, jack and tuna [see Parrish \& Edelstein-Keshet (1999) for a photograph of this behaviour in jack]. 
(A)
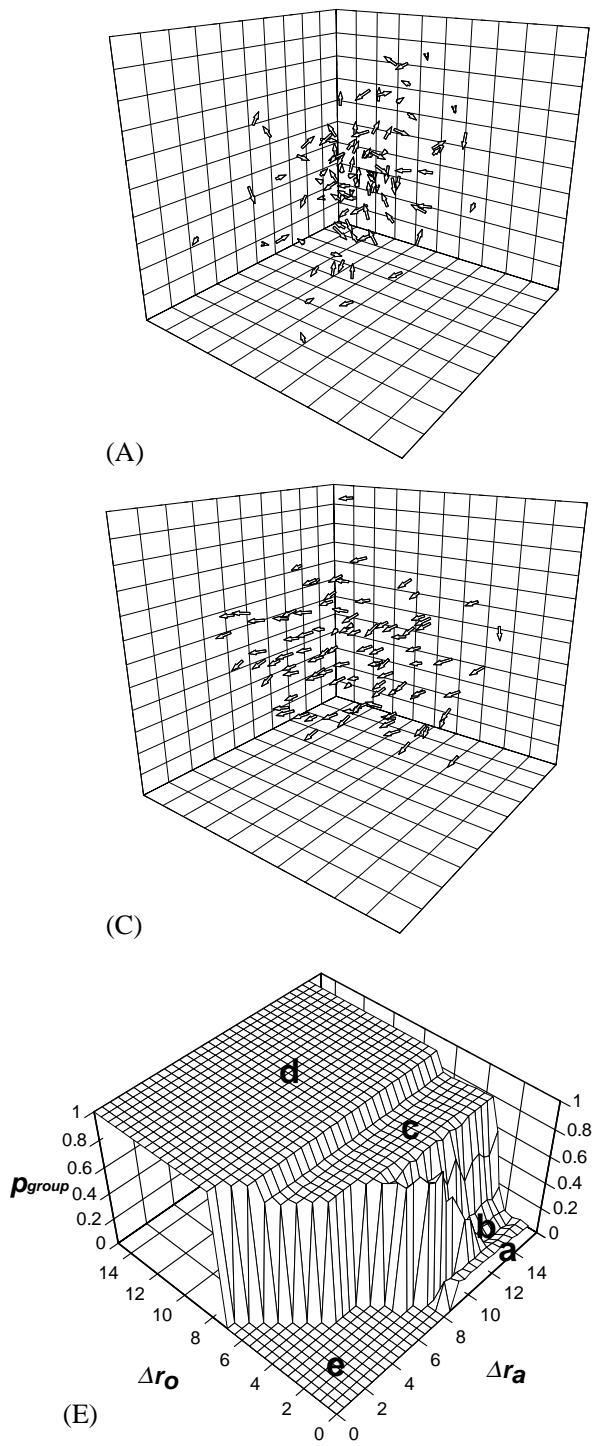

(B)
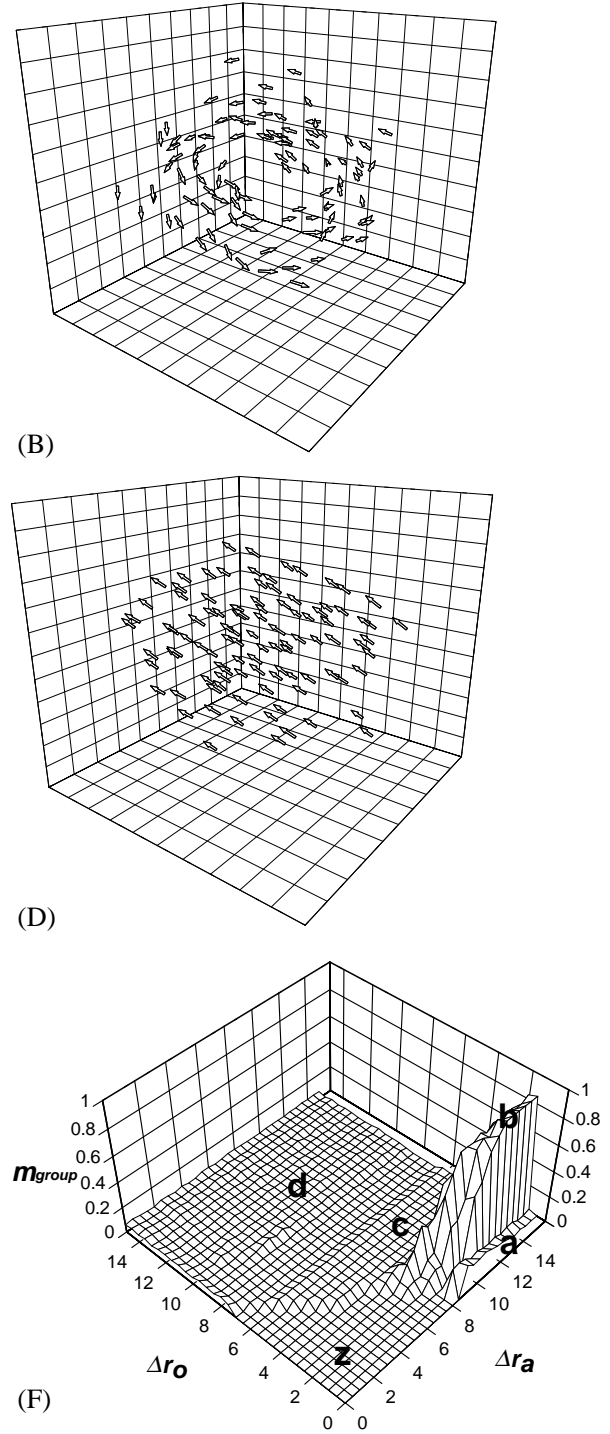

Fig. 3. The collective behaviours exhibited by the model: (A) swarm, (B) torus, (C) dynamic parallel group, (D) highly parallel group. Also shown are the group polarization $p_{\text {group }}(\mathrm{E})$ and angular momentum $m_{\text {group }}(\mathrm{F})$ as a function of changes in the size of the zone of orientation $\Delta r_{o}$ and zone of attraction $\Delta r_{a}$. The areas denoted as (a-d), correspond to the area of parameter space in which the collective behaviours (A-D), respectively, are found. Area (e) corresponds to the region in parameter space, where groups have a greater than $50 \%$ chance of fragmenting. $N=100, r_{r}=1, \alpha=270, \theta=40, s=3$, $\sigma=0.05$. Data shown in (E) and (F) are the mean of 30 replicates per parameter combination.

Fig. 3(E) and (F) show the group polarization $p_{\text {group }}$ and angular momentum $m_{\text {group }}$, respectively, as $\Delta r_{o}$ and $\Delta r_{a}$ vary. The area of zero values when $\Delta r_{o}$ and $\Delta r_{a}$ are relatively low [Fig. 3(E) and (F), region e] corresponds to the area of parameter space, where groups have a greater than $50 \%$ chance of fragmenting. Since the collective behaviour is dependent on group size, analysis is only performed on non-fragmented groups. The group types shown in Fig. 3 exist for all group sizes analysed, although the range over which the torus and dynamic parallel groups form tends to decrease as the group size decreases. The field of perception also influences the collective behaviour. The range in which groups form a torus is diminished to a very small range of $\Delta r_{o}$ and $\Delta r_{a}$ when the field of perception, $\alpha$, is $360^{\circ}$, but increases as $\alpha$ decreases. Parallel groups become more elongated along their principal axis (the direction of 
travel) as $\alpha$ diminishes, and the probability that they will fragment increases. When $\alpha$ reaches approximately $230^{\circ}$ group fragmentation is common across the entire parameter space, and as it decreases further it becomes less likely that groups will remain cohesive.

The turning rate of individuals essentially rescales the parameter space; increasing the rate of turning "compresses" parameter space allowing the collective patterns to form with relatively smaller values of $\Delta r_{o}$ and $\Delta r_{a}$, whereas decreasing the rate of turning increases the area in which groups fragment and increases the $\Delta r_{o}$ and $\Delta r_{a}$ at which the groups enter the behavioural states (i.e. it "expands" the parameter space). Speed has a similar influence to turning, since it modifies the turning arc of individuals (higher speed increases the size of the turning arc, as does decreasing turning rate). If the noise parameter is close to zero, the swarm and torus area of parameter space remain largely unchanged, but the area in which groups form dynamic parallel groups is reduced, with highly parallel groups forming at relatively low $\Delta r_{o}$ values. As noise is increased, the dynamic parallel area first expands in the $\Delta r_{o}$ dimension and then individual error becomes too high for polarized, or torus group types to form, and swarm-like behaviour persists over much of the parameter space. As noise increases, there is also a higher probability that groups will fragment, so the values of $\Delta r_{o}$ and $\Delta r_{a}$ at which stable groups form tends to increase.

\section{HYSTERESIS AND BEHAVIOURAL TRANSITIONS}

In the above analysis, the starting orientations of individuals are always random. However, in nature, groups are likely to move between collective states as conditions change; consequently the previous history of individual orientation and group shape may influence future collective behaviour as behavioural parameters change. By changing the way they respond to others, individuals can change the structure of the group to which they belong.

To investigate this process, we explored how changes in individual behaviour result in a change of the collective behavioural state. Figure 4 shows how $p_{\text {group }}$ and $m_{\text {group }}$ change as
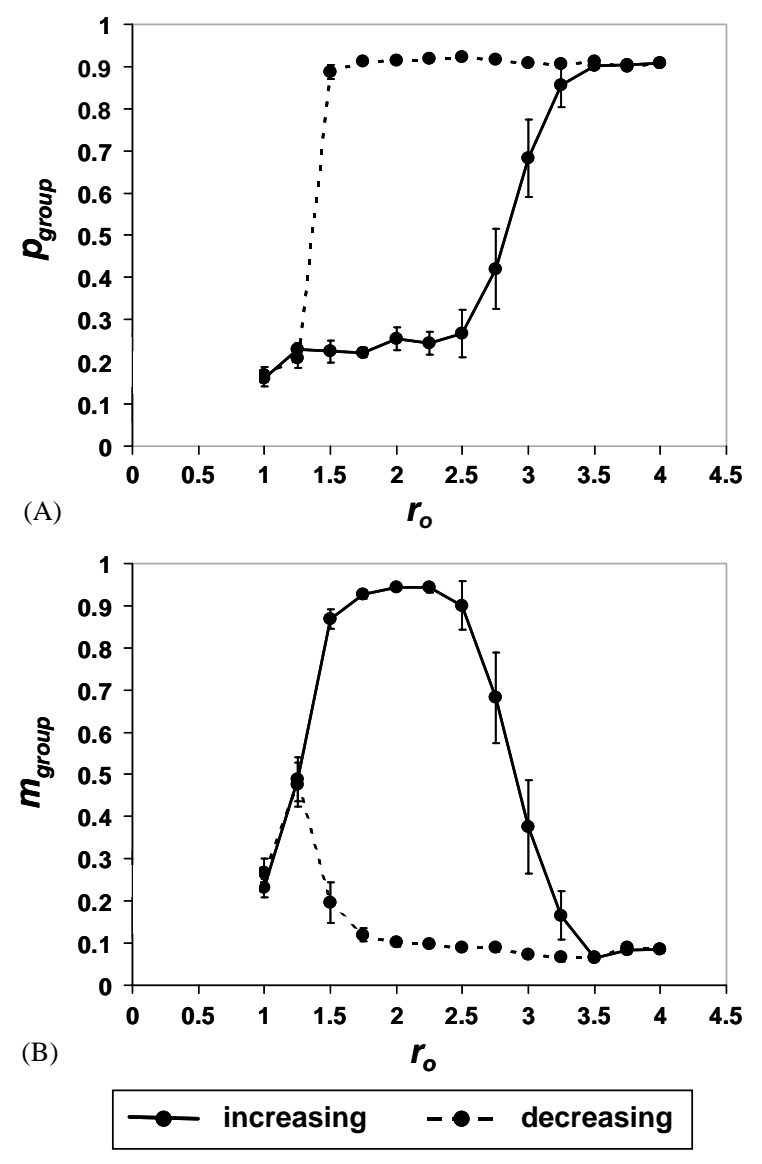

FIG. 4. The change in group polarization $p_{\text {group }}(\mathrm{A})$ and angular momentum $m_{\text {group }}(\mathrm{B})$ as individuals within a group increase (bold line) or decrease (dotted line) the size of the zone of orientation $r_{o}$. Two thousand time steps were run under each value of $r_{o}$ before it was incremented or decremented. Fifteen replicates were performed, and the average value plotted with error bars, indicating the standard error. The group patterns that form depend on the previous history of the group (hysteresis). Parameters as for Fig. 3, $r_{a}=14$.

individuals within the group modify their response to others by keeping $r_{a}$ fixed and either increasing, or decreasing their $r_{o}$ over a given range, resulting in the group changing between swarm, torus and dynamic parallel collective behaviours. If $r_{o}$ is close to $1\left(\Delta r_{o} \approx 0\right)$, the group exhibits swarm behaviour (low $p_{\text {group }}$, low $\left.m_{\text {group }}\right)$. As $r_{o}$ increases towards 1.5 , the group exhibits a rapid behavioural transition and enters the torus phase ( $m_{\text {group }}$ rapidly increases). Beyond 2.5, however, the group enters a polarized configuration $\left(m_{\text {group }}\right.$ decreases and $p_{\text {group }}$ increases). However, if the group moves back through parameter space in the 
opposite direction, decreasing $r_{o}$, we show that the group structure is different. Group polarization does not decrease, and angular momentum remains low and the group does not adopt the torus conformation. As $r_{o}$ decreases below 1.5, however, the polarization rapidly disappears and the group readopts the swarm configuration. This shows that the transition between behavioural states depends on the previous history (structure) of the group, even though individuals have no explicit knowledge of what that history is.

\section{SELF-SORTING}

Differences in individual movement patterns, or behavioural motivations, influence the spatial positions occupied within a group (Fig. 5). Differences in speed $s$, turning rate $\theta, r_{r}$, and $r_{o}$ all influence the distribution of individuals relative to either, or both, the centre and front of groups [Fig. 5(A)-(D)], whereas error $\sigma$ influences only that from the front. $r_{a}$ has no significant effect. Speed is strongly positively correlated with being at the front of the group, showing that faster individuals tend to occupy positions near the front of moving groups. Faster individuals also tend to be further from (negatively correlated with) the group centre, although this correlation is less strong [Fig. 5(A)]. Turning rate is negatively correlated with being at the front of the group, and slightly positively correlated with being at the group centre, demonstrating that individuals with a higher rate of turning tend to be at the rear, and slightly closer to the centre of the group, than are individuals with a lower rate of turning [Fig. 5(B)]. The degree of error in individual movement is negatively correlated only with being at the front of the group, with individuals exhibiting higher error occupying positions towards the rear of the group.

Only variation in the zone of repulsion, $r_{r}$, and zone of orientation, $r_{o}$, influence sorting within
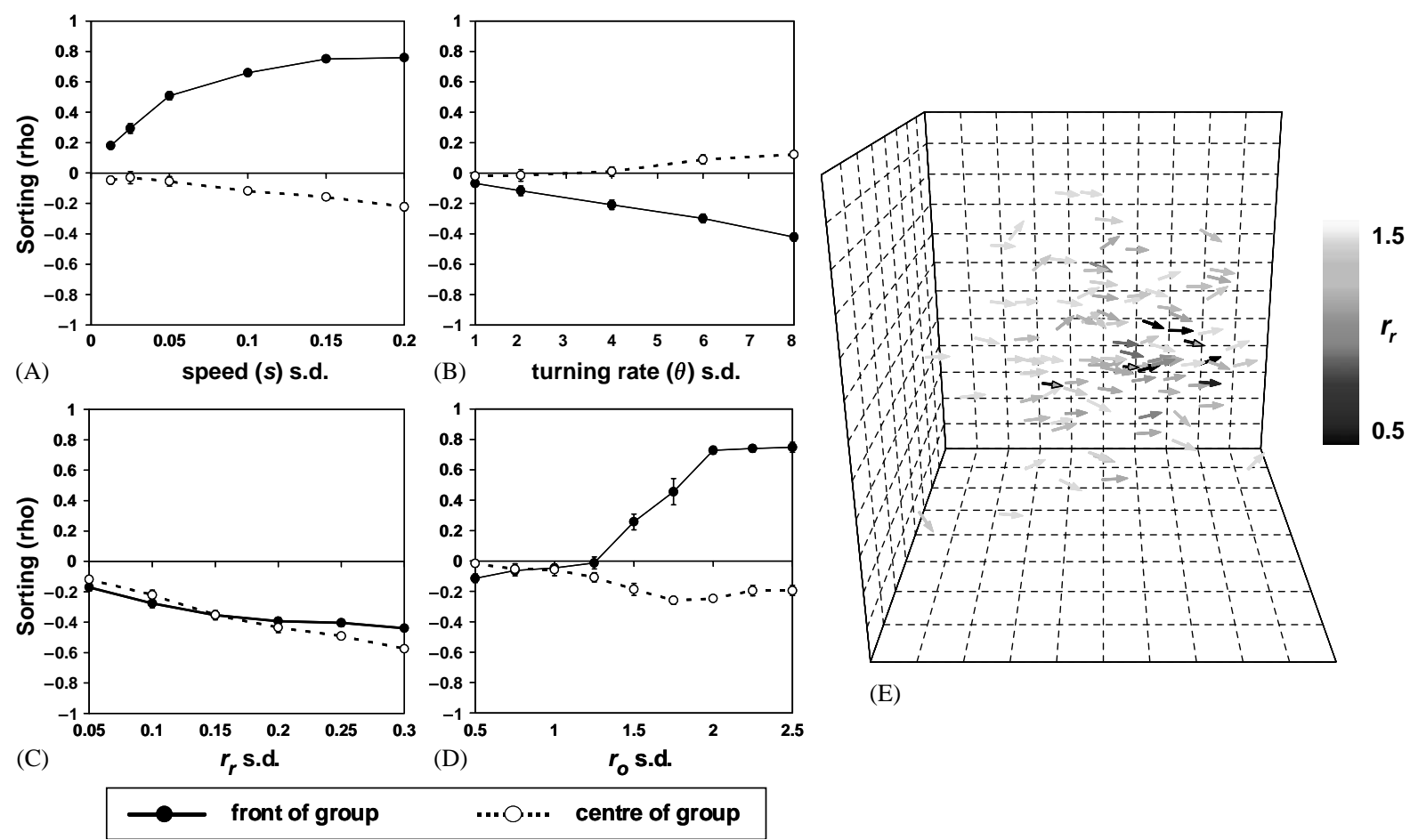

(E)

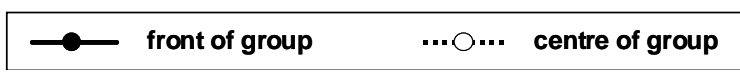

FIG. 5. Sorting as a function of variation in (A) speed $s$, (B) turning rate $\theta$, (C) zone of repulsion $r_{r}$, and (D) zone of orientation $r_{o}$. A typical group sorted by $r_{r}$ is shown in (E). Parameters as for Fig. 3, with the exception of the sorting parameter. $r_{o}=6, r_{a}=14$. Data shown are the mean of 30 replicates per parameter combination, and error bars indicate the standard error. Sorting is measured as the Spearman rank correlation coefficient (rho) of individuals calculated from the front (solid line) or centre (dotted line) of the group. 
groups [Fig. 5(C) and (D), respectively], with variation in the zone of attraction, $r_{a}$, having no effect over the range of parameters investigated. There is a negative correlation between both the radius of the zor and being at the front or the centre of the group. This shows that individuals with smaller $r_{r}$ values tend to be closer to the centre and front of the group than do individuals with larger $r_{r}$ [Fig. 5(C) and (E)]. The radius of the zone of orientation, $r_{o}$, is very slightly negatively correlated with being at the front of the group at very low levels of variance, but becomes strongly positively correlated as the variance increases. Intuitively, the increase in orientation response could be expected to positively correlate with being in frontal positions since such individuals have a larger range over which they align to others, and their movement consequently tends to exhibit lower deviations in angle from the direction of travel than individuals with a smaller $r_{o}$. However, currently, we cannot explain the very slight negative correlation between $r_{o}$ and being at the front of the group when variation in $r_{o}$ is very low.

For all parameters where a correlation is found, the strength of the correlation tends to increase as the variation among individuals increases.

\section{Discussion}

Our model exhibits several collective behaviours, with sharp transitions between them (see also Suzuki \& Sakai, 1973 and Shimoyama et al., 1996). Small changes in individual responses result in large changes in group properties and organization. The model predicts that animal groups, such as fish schools, change rapidly between these states (for example, between the torus and dynamic parallel group types) since intermediate group types are relatively (dynamically) unstable. Biologically the transitions are important in allowing animal groups to change from one type of group structure to another in response to internal (e.g. hunger) or external (e.g. detection of a predator) stimuli, as individuals attempt to maximize their fitness as circumstances change. The tendency of individuals to align with one another within the parallel group types is important not only in minimizing collision between individuals and facilitating group movement, but also in allowing the group to transfer information. For example, if an individual were to turn sharply, as a response to avoiding a predator or an obstacle, the alignment tendency allows this turn to influence the orientation of neighbours (which need not directly detect the stimulus), facilitating a transfer of information (turning) over a range greater than the individual interaction radius. If individuals exhibit attraction, but little or no alignment to neighbours, they exhibit the swarm group type. This group is very cohesive, but the relatively high variance of individual orientation means that the change in direction of group members, as a result of detecting a predator for example, is propagated less efficiently. The torus group type may also benefit from the local (although not global) polarization of individuals in allowing a group to remain relatively stationary (which may be beneficial when resting, or to avoid encounters with sit-and-wait predators) yet still facilitates information transmission. This group type exhibits continual motion (needed by certain species of fish for respiration), and may provide energetic savings as a result of individuals using the slipstreams of others.

As well as demonstrating that collective behavioural transitions occur in our model, we also investigated the way such transitions may occur in natural animal groups as a result of individuals modifying their interactions with one another. Intuitively, one may assume that group-living animals need only evolve a direct relationship between internal state (resulting from internal and external stimuli) and behaviour (here the rules of interaction employed). However, our model reveals that two very different collective states can occur for identical individual behaviour, demonstrating the importance of the previous history of the group structure. This hysteresis phenomenon is an important result, because it suggests that the evolution of individual behavioural responses to facilitate collective transitions may be more complex than it would originally appear. Understanding how and why animal groups change from one collective state to 
another is essential if we are to understand the evolution of animal grouping. Such collective memory may be an as yet undiscovered property of group behaviour transitions in animal groups such as fish schools. This prediction could be directly tested for fish species that exhibit both the torus and dynamic parallel group structures. Furthermore, this type of phenomenon may exist during transitions between collective behaviours in other animal groups as individuals change their response to others.

We also used the model to investigate mechanisms underlying the generation and maintenance of spatial positioning within animal groups. The results of the model suggest that behavioural and/or motivational differences between organisms may have an important structuring influence when animals aggregate. Individuals may change their position relative to others within groups based upon internal state such as satiation level, or of perceived risk of predation. For example, Krause (1993) in a study of schooling fish (roach, Rutilus rutilus), demonstrated that starved individuals will tend to take positions near the front of the school more often than will those that are satiated. This is likely to maximize the resource intake by such individuals, but may also put them at increased risk of attack by predators (Bumann et al., 1997). The model demonstrates several potential self-organizing mechanisms ("rules of thumb") by which individuals can modify their position within groups. This sorting depends not on the absolute values of the parameters, but rather on the relative difference between individuals. For example, if an individual decreases the size of the local area of repulsion relative to those around them, it will move towards the centre and front of the group, even if it has no knowledge of where it is within the group. This is important because it is unlikely that organisms, such as pelagic fish within large schools, have the cognitive or sensory capabilities to determine their location within their group (which may consist of hundreds of thousands, or even millions of individuals) and then adjust their position relative to that. There may also be limits to the information available to grouping individuals. For example, the average distance main- tained between neighbours within pelagic fish schools is usually between three-tenths of a body length and one body length (Partridge, 1982), and it is extremely unlikely that individuals inside the group are aware of their position relative to the front, or centre. Thus, natural selection is likely to favour mechanisms such that individuals can change their position relative to others based only on local information. Another property of a system self-sorted in this way is that if individual differences in behaviour are intrinsic, or tend to be consistent, the system will reassemble forming (statistically) the same configuration after perturbation from that state. A generic result of this sorting process is that individuals with similar behaviours tend to become aggregated within the group. In many fish schools, individuals tend to be close to other individuals of a similar size (Pitcher et al., 1985; Parrish, 1989; DeBlois \& Rose, 1996). This mechanism may also underlie the sorting behaviour within multispecies schools, where individuals have been found to assort by both species and size (Parrish, 1989). The results of our model suggest that if size and/or species is correlated with the behavioural response, or the movement properties of fish, that this could account for the assortment seen. Modelling self-sorting may also be valuable in understanding how parasites manipulate the positions of individuals within groups. For example, Krause \& Godin (1994) found that banded killifish parasitized with Crassiphiala bulboglossa, a trematode worm, will tend to occupy peripheral positions within a shoal of unparasitized conspecifics (see also Barber \& Huntingford, 1996 for a similar hostparasite system). They suggest that the parasite may have evolved the ability to manipulate the host behaviour. Our model provides potential mechanisms of sorting that may help experimentalists to better understand what behavioural modification occur in such parasitized individuals.

I.D.C would like to thank Nick Davies, Stuart Reynolds and Rob Sheichl for helpful discussions, and the BBC Natural History Unit for their support. I.D.C. was funded by NERC and The Leverhulme Trust (F/122/BD). 


\section{REFERENCES}

AoKI, I. (1982). A simulation study on the schooling mechanism in fish. Bull. Jap. Soc. Sci. Fish. 48, 1081-1088.

Barber, I. \& Huntingford, F. A. (1996). Parasite infection alters schooling behaviour: deviant positioning of helminth-infected minnows in conspecific groups. Proc. R. Soc. Lond. Ser. B 263, 1095-1102.

Bumann.D., Krause, J. \& Rubenstein, D. I. (1997). Mortality risk of spatial position in animal groups: the danger of being in the front. Behaviour 134, 1063-1076.

Czirók, A., Stanley, H. E. \& Vicsec, T. (1997). Spontaneously ordered motion of self-propelled particles. J. Phys. A 30, 1375-1385.

Czirók, A., Vicsek, M. \& Vicsek, T. (1999). Collective motion of organisms in three dimensions. Physica A 264, 299-304.

CZirók, A. \& VicseK, T. (2001). Collective motion. In: Fluctuations and Scaling in Biology (Vicsek, T., ed.), pp. 177-242. Oxford: Oxford University Press.

Deblois, E. M. \& Rose, G. A. (1996). Cross shoal variability in the feeding habits of migrating Atlantic cod (Gadus morhua). Oecologia 108, 192-196.

Gueron, S., Levin, S. A. \& Rubenstein, D. I. (1996). The dynamics of herds: from individuals to aggregations. J. theor. Biol. 182, 85-98.

Helbing, D., Farkas, I. \& Vicsek, T. (2000). Simulating dynamical features of escape panic. Nature 407, 487-490.

Heppner, F. H. (1997). Three-dimensional structure and dynamics of birds flocks. In: Animal Groups in Three Dimensions (Parrish, J. K. \& Hamner, W. M. eds). Cambridge: Cambridge University Press.

Huth, A. \& Wissel, C. (1992). The simulation of the movement of fish schools. J. theor. Biol. 156, 365-385.

IKaWA, T. \& OKaBE, H. (1997). Three-dimensional measurements of swarming mosquitoes: a probabilistic model, measuring system, and example results. In: Animal Groups in Three Dimensions (Parrish, J. K. \& Hamner, W. M., eds). Cambridge: Cambridge University Press.

Krause, J. (1993). The relationship between foraging and shoal position in a mixed shoal of roach (Rutilus rutilus) and chub (Leuciscus cephalus): a field study. Oecologia 93, 356-359.

Krause, J. (1994). Differential fitness returns to spatial positions in groups. Biol. Rev. 69, 187-206.

Krause, J. \& Godin, J.-G. J. (1994). The influence of parasitism on the shoaling behaviour of the killifish (Fundulus diaphanus). Can. J. Zool. 72, 1775-1779.
Krause, J, Godin, J.-G. J. \& Brown, D. (1996). Phenotypic variability within and between fish shoals. Ecology 77, 1586-1591.

Krause, J. \& Ruxton, G. D. (2002). Living in Groups. Oxford: Oxford University Press.

Major, P. F. \& Dill, L. M. (1978). The three-dimensional structure of airborne bird flocks. Behav. Ecol. Sociobiol. 4, 111-122.

Oкuво, A. (1980). Diffusion and Ecological Problems: Mathematical Models, Lecture Notes in Biomathematics, Vol. 10. New York: Springer-Verlag.

Oкubo, A. \& Chiang, H. C. (1974). An analysis of the kinematics of swarming behavior of Anarete pritchardi Kim (Diptera: Cecidomyiidae). Res. Popul. Ecol. 16, $1-42$.

PARrish, J. K. (1989). Layering with depth in a heterospecific fish aggregation. Env. Biol. Fishes 26, 79-86.

Parrish, J. K. \& Edelstein-Keshet, L. (1999). Complexity, pattern, and evolutionary trade-offs in animal aggregation. Science 284, 99-101.

PARTRIDGE, B. L. (1982). The structure and function of fish schools. Sci. Am. 245, 90-99.

Partridge B. L. \& Pitcher T. J. (1980). The sensory basis of fish schools: relative role of lateral line and vision. J. Comparative Physiol. 135, 315-325.

Pitcher, T. J. \& Parrish, J. K. (1993). The functions of shoaling behavior. In: The Behavior of Teleost Fishes (Pitcher, T. J. ed.), pp. 363-439. London: Chapman \& Hall.

Pitcher, T. J., Magurran, A. E. \& Edwards, J. I. (1985). Schooling mackerel and herring choose neighbours of similar size. Mar. Biol. 86, 319-322.

Press, W. H., Teukolsky, W. A., Vetterling, W. T. \& Flannery, B. P. (1992). Numerical Recipes in $C$ : the Art of Scientific Computing, 2nd Edn. Cambridge: Cambridge University Press.

ReYnOLDs, C. W. (1987). Flocks, herds and schools: a distributed behavioral model. Comput. Graph. 21, 25-34.

Shimoyama, N., Sugawara, K., Mitzuguchi, T., HayaKAWA, Y. \& SANO, M. (1996). Collective motion in a system of motile elements. Phys. Rev. Lett. 76, 3870-3873.

Suzuki, R. \& SaKaI, S. (1973). Movement of a group of animals. Biophysics 13, 281-282.

WARBURTON, K. (1997). Social forces in animal congregations: interactive, motivational, and sensory aspects. In: Animal Groups in Three Dimensions. (Parrish, J. K. \& Hamner, W. M., eds), pp. 313-333. Cambridge: Cambridge University Press. 